Article

\title{
Green Synthesis of Silver Nanoparticles by Low-Energy Wet Bead Milling of Metal Spheres
}

\author{
Andrea Pietro Reverberi ${ }^{1, *}$, Marco Vocciante ${ }^{1}\left(\mathbb{D}\right.$, Marco Salerno $^{2}\left(0\right.$, Maurizio Ferretti $^{1}(\mathbb{C})$ and \\ Bruno Fabiano $^{3}$ \\ 1 DCCI, Department of Chemistry and Industrial Chemistry, Università degli Studi di Genova, \\ Via Dodecaneso 31, 16146 Genova, Italy; marco.vocciante@gmail.com (M.V.); ferretti@chimica.unige.it (M.F.) \\ 2 Nanophysics Department, Istituto Italiano di Tecnologia, via Morego 30, 16163 Genova, Italy; \\ marco.salerno@iit.it \\ 3 DICCA, Department of Civil, Chemical and Environmental Engineering, Polytechnic School, \\ Università degli Studi di Genova, Via Opera Pia 15, 16145 Genova, Italy; brown@unige.it \\ * Correspondence: reverb@dichep.unige.it
}

Received: 16 November 2019; Accepted: 19 December 2019; Published: 21 December 2019

check for updates

\begin{abstract}
A low-energy, magnetically-driven milling technique for the synthesis of silver nanoparticles is proposed, where the grinding medium and the metal precursor consisting of silver spheres have the same shape and size, belonging to a millimetric scale. The process is carried out at room temperature in aqueous solvent, where different types of capping agents have been dissolved to damp particle agglomeration. The particle diameters, determined by dynamic light scattering and transmission electron microscopy, have been compared with those typical of conventional wet-chemical bottom-up synthesis processes. The use of milling spheres and metal precursor of the same initial shape and size allows to overcome some drawbacks and limitations distinctive of conventional bead-milling equipment, generally requiring complex operations of separation and recovery of milling media. The milling bead/nanoparticle diameter ratio obtained by this approach is higher than that typical of most previous wet bead milling techniques. The method described here represents a simple, one-pot, cost-effective, and eco-friendly process for the synthesis of metal nanoparticles starting from a bulky solid.
\end{abstract}

Keywords: nanoparticle synthesis; bead milling; top-down method; eco-friendly process; green nanotechnology

\section{Introduction}

In recent years, the methods for the synthesis of nanoparticles (NPs) have received progressively growing attention and many techniques have been refined according to the physico-chemical properties of the specific NPs, as indicated in the scheme reported in Figure 1. Many manufacturing processes have been improved and implemented on large-scale facilities, as in the production of nanosized alumina, zirconia, and other inorganic compounds like ceramic materials [1]. In other cases, the processes are still limited to a smaller scale, typical of pilot plants or even a laboratory environment [2], owing to constraints related to safety [3], lack of process control, and cost of unit operations. 


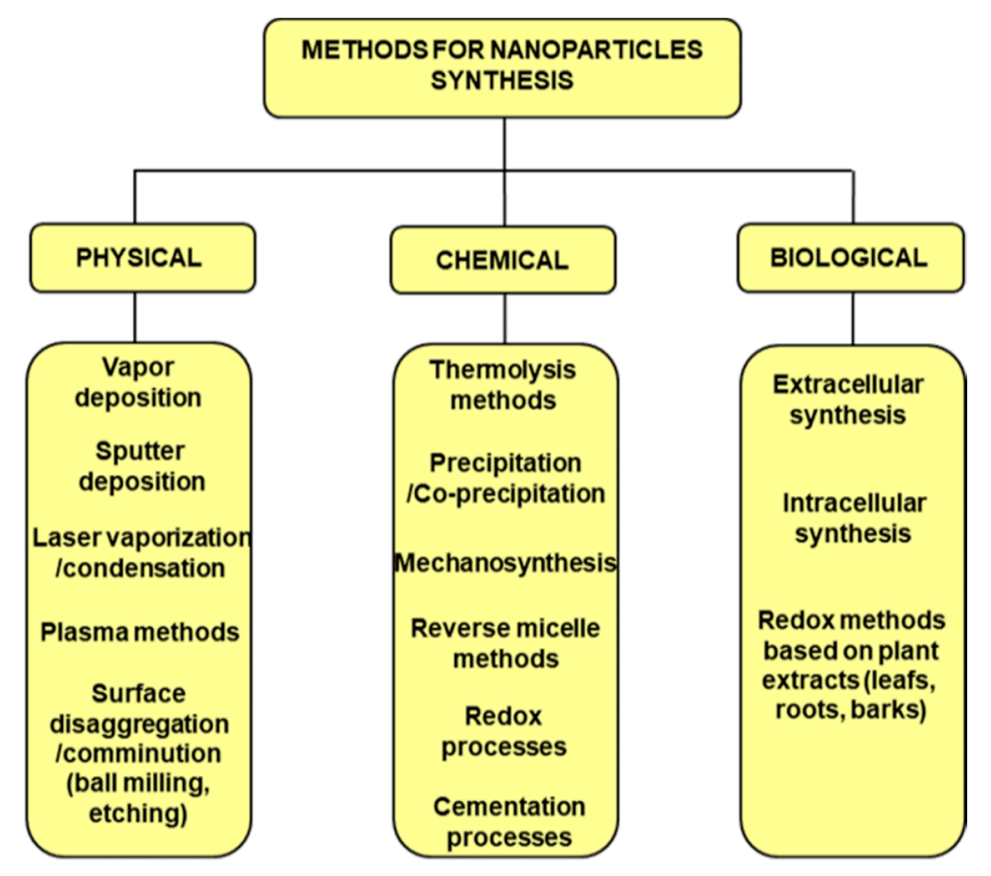

Figure 1. Scheme of the main methods adopted for nanoparticles (NPs) synthesis.

Very often, chemical methods are a first-rank choice in several synthesis techniques concerning the production of nanosorbents for environmental remediation [4], the preparation of nitrogen-doped graphene (NG) as a metal-free carbon-based catalyst to degrade recalcitrant organics [5], the synthesis of nanoceramics for application in dentistry [6], the fabrication of nanosized sensors to ensure safety conditions in oxidation processes [7], and the production of zerovalent NPs by redox processes [8]. The latter are generally carried out in liquid phases [9], where a precursor containing the ion of the element forming the nanoparticles is dissolved and mixed with a proper electron-donor carrying out the reduction process. An exhaustive discussion about redox methods for the production of NPs for weak and strong electropositive cations can be found in the review papers of Daruich De Sousa et al. [10] and Stefaniuk et al. [11], respectively, and we refer the reader to the references quoted there. The main drawback related to the aforementioned bottom-up redox methods depends on the use of organic or inorganic reductants with toxic effects on humans and the environment. The paper of Rodrigues et al. [12] is a very exhaustive survey concerning a wide choice of electron-donors used in metal nanocrystal synthesis. Among them, hydrazine and its compounds, despite its excellent properties as a reducing agent, joined with a relatively low cost are subject to safety restrictions for their cytotoxic, mutagen, and carcinogenic activity, both on microorganisms and vertebrates [13]. For these reasons, a strategy based on reagent substitution protocols is becoming a paradigm in eco-friendly NPs synthesis [14], as proven by the recent development of green methods based on reagents and capping agents of biological origin [15] and by the overall trend towards the neologistic "green nanotechnology" [16]. As an example, this aspect finds important applications in heat transfer technology [17], where nanofluids as heat carriers need to combine a low toxicity with an efficient stabilization against agglomeration for a wide range of temperature and fluid-dynamics conditions [18]. Nowadays, many reagents derived from plant extracts, pigments, yeast, and enzymes have been used as reductants for the synthesis of zerovalent NPs [19], with more positive results in cases of precursors containing noble metals like gold, platinum, palladium, silver [20], and other elements of weakly electropositive properties [21]. Top-down chemical methods, though representing a cost-effective alternative strategy for the production of inorganic nanoparticles, are still confined to a narrow field, probably owing to a lack of control on particle diameters, whose broad distribution adversely affects 
the quality of the final product. In some cases, a combination of top-down etching methods with traditional bottom-up routes led to promising results in surface patterning and functionalization [22].

A further step forward in the minimization of environmental impact of NPs manufacturing is offered by top-down physical methods [23], in which a nanomaterial is formed starting from elements or compounds in macroscopic sizes undergoing treatments without any chemical reaction. Several disaggregation techniques have been proposed [24], whose classification generally depends on the presence/absence of milling media like spheres or beads in a vessel containing the phase subject to comminution [25]. The effect of mechanical impact and friction may be advantageously controlled by the use of proper capping agents damping the simultaneous reaggregation tendency of the as-produced nanoparticles [26]. In some cases, as in cryomilling processes, the presence of such stabilizing agents is avoided in order to obtain a nanophase totally free of surface contaminants [27].

The present work belongs to the context of top-down physical methods, in that it deals with the formation of nanosized particles of zerovalent Ag through disaggregation of silver spheres, whose surface is subject to impact and abrasion carried out by ceramic balls of yttrium-stabilized zirconia dispersed in aqueous solvent where capping agents have been previously dissolved. The choice of the aforementioned grinding medium is motivated by its high density, negligible surface porosity, and peculiarity of having an increasing tenacity with a growing number of collision events. The presence of a small concentration of $\mathrm{Y}_{2} \mathrm{O}_{3}$ has a limited effect on the cost of such ceramic balls.

Essentially, the main purpose of this work is to create a dispersion of Ag NPs in a one-pot process, using both a metal precursor $(\mathrm{Ag})$ and a milling medium with diameters at a millimetric scale. This strategy may represent an alternative to conventional wet bead millers, which are generally designed to break NPs agglomerates as a source material, rather than producing NPs starting from a bulky phase, as in the case proposed here. The paper is divided as follows. In Section 2, the process of synthesis is described and some details concerning the experimental set-up are given. In Section 3, the results are presented and related to the previous outcomes in literature concerning wet disaggregation techniques. In Section 4, the conclusions are drawn and the direction for future work is traced.

\section{Materials and Methods}

\subsection{Composition of the Vessel Content}

Milli-Q water was used as solvent in all experimental samples.

Silver spheres (Ag, 99.9\%, American Elements, Los Angeles, CA, USA) were adopted as metal precursor undergoing disaggregation. Yttria-stabilized zirconia spheres $\left(\mathrm{ZrO}_{2} 95 \%, \mathrm{Y}_{2} \mathrm{O}_{3} 5 \%, \mathrm{MSE}\right.$ Supplies, Tucson, AZ, USA) were used as supplied.

The list of capping agent adopted here comprises urea (UR, $\mathrm{CO}\left(\mathrm{NH}_{2}\right)_{2}, 99 \%$, La Farmochimica, Genova, Italy); tetrabutylammonium bromide ( $\mathrm{TBAB},\left(\mathrm{C}_{4} \mathrm{H}_{9}\right)_{4} \mathrm{NBr}, 99 \%$, Merck, Milano, Italy); sodium dodecyl sulphate (SDS, $\mathrm{CH}_{3}\left(\mathrm{CH}_{2}\right)_{11} \mathrm{OSO}_{3} \mathrm{Na}$, 99\%, Sigma-Aldrich, Milano, Italy); $\mathrm{N}$,N-dimethyldodecylamine $\mathrm{N}$-oxide (DDAO, $\mathrm{CH}_{3}\left(\mathrm{CH}_{2}\right)_{11} \mathrm{NO}\left(\mathrm{CH}_{3}\right)_{2}, 30 \%$ in water, Sigma-Aldrich, Milano, Italy); and three different forms of polyvinyl pyrrolydone (PVP, $\left(\mathrm{C}_{6} \mathrm{H}_{9} \mathrm{NO}\right)_{\mathrm{n}}, 99 \%$, Sigma-Aldrich, Milano, Italy), with $10 \mathrm{kDa}, 40 \mathrm{kDa}$, and $750 \mathrm{kDa}$ as average molecular weights.

In the following, the experimental samples are labelled with letters $a-g$ according to the type of capping agent adopted, as reported in Table 1. PVP was chosen in three different compositions to ascertain the effect of its different molecular weights on the final product. The concentration of capping agents for samples $a, b, c$ was fixed at $0.2 \mathrm{~g}$ in $3 \mathrm{~cm}^{3}$ of liquid hold-up, in order to ensure the same number of monomers for each sample. For samples $d, e, f, 0.1 \mathrm{~g}$ in $3 \mathrm{~cm}^{3}$ of liquid hold-up was set. For sample $g$, the concentration was $0.3 \mathrm{~cm}^{3}$ in $3 \mathrm{~cm}^{3}$ of water. 
Table 1. List of experimental samples and their composition. PVP, polyvinyl pyrrolydone; UR, urea; TBAB, tetrabutylammonium bromide; SDS, sodium dodecyl sulphate; DDAO, $\mathrm{N}, \mathrm{N}$-dimethyldodecylamine $\mathrm{N}$-oxide.

\begin{tabular}{ccc}
\hline Sample Label & Capping Agent & Type of Capping Agent \\
\hline$a$ & PVP 10k & Non ionic \\
$b$ & PVP 40k & Non ionic \\
$c$ & PVP 750k & Non ionic \\
$d$ & UR & Non ionic \\
$e$ & TBAB & Cationic \\
$f$ & SDS & Anionic \\
$g$ & DDAO & Zwitterionic \\
\hline
\end{tabular}

\subsection{The Experimental Set-up}

The home-made stirring/milling equipment comprises a round bottom cylindrical vessel of $13.5 \mathrm{~cm}^{3}$ volume, where a polytetrafluoro ethylene (PTFE)-coated magnetic stirring bar, whose length is greater than the inner diameter of the vessel, is forced to rotate in a skew position with respect to the axis $a b$ of the vessel by means of a magnetic stirrer, located at the bottom of the container schematically represented in Figure 2a. In each experimental sample, besides the stirring bar (15 mm length; $4.5 \mathrm{~mm}$ diameter), the vessel contains the following:

- $\quad 3 \mathrm{~cm}^{3}$ of solvent, where the corresponding mass of capping agent was previously dissolved;

- $\quad 40 \mathrm{ZrO}_{2} / \mathrm{Y}_{2} \mathrm{O}_{3}$ ceramic spheres of $3 \mathrm{~mm}$ diameter, forming a bed in static conditions whose height must allow the rotation of the stirrer without jams;

- $\quad$ three silver spheres of $3 \mathrm{~mm}$ diameter.

There is not a common opinion about the maximum allowed diameter of grinding spheres in a bead milling process, but a diameter of $3 \mathrm{~mm}$ adopted in the present study can be considered as an upper threshold value [28].

Two cylindrical magnets, positioned on a disk below the flat surface of the stirrer in Figure 2b, turn around the axis $c d$ with adjustable speed, which was maintained at $600 \mathrm{rpm}$ in all experiments.

A considerable lack of milling efficiency was observed when the axes $a b$ and $c d$, drawn as dashed lines in Figure 2b, overlap one another. This phenomenon can be ascribed to a drop of frequency of collisional events between spheres, irrespective of their composition. For this reason, in the present experimental campaign, the axis $a b$ was shifted by approximately $5 \mathrm{~mm}$ in parallel to the axis $c d$.

The vessel is gas-tight to avoid the evaporation of the solvent, but the process is carried out in the presence of air. On the opposite, previous studies showed that the use of inert gases is necessary when zerovalent NPs of non-noble metals are produced, owing to their high tendency for oxidation [29]. Unlike many high-energy milling processes, no noticeable temperature rise was detected here during the milling process, and hence no cooling equipment was necessary. After $5 \mathrm{~h}$ of milling time for all experimental samples, the liquid hold-up is allowed to stand for $12 \mathrm{~h}$ and it is further centrifuged at $8000 \mathrm{rpm}$ for $15 \mathrm{~min}$. The supernatant is then separated and analyzed with the instruments described in the next section.

\subsection{Characterization Techniques}

The average hydrodynamic size of the fabricated Ag NPs was obtained by carrying out dynamic light scattering (DLS) in the surfactant-modified aqueous media of the respective samples. We used a Zetasizer Nano instrument (Malvern, UK), equipped with a red light source. The cuvettes were disposable polystyrene (VWR, Leuven, Belgium), $2.5 \mathrm{~mL}$ in size, filled in with approximately $1.2 \mathrm{~mL}$ suspension. The acquisition was run in non-invasive backscattering mode $\left(173^{\circ}\right)$, typically based on three measurements of seven runs each (run duration $10 \mathrm{~s}$, no delay in between), started after $120 \mathrm{~s}$ sample equilibration time at $25^{\circ} \mathrm{C}$. We used the general purpose model of time autocorrelation in the 
Zetasizer V.7.11 software (no protein analysis). For the optical properties of the Ag NPs, we used a refractive index of 0.2 and an absorption coefficient of 0.6 . Given the little amount of surfactants, the dispersant was always modeled as pure water, with a refractive index of 1.33 and viscosity of 0.8872 cP. After running the measurement, the raw data of size distribution by scattered light intensity are automatically converted-via the built-in model—to size distribution by volume, and finally to size distribution by number (\%), which is what is reported in the figures. The absolute count rate was always in the order of $200 \mathrm{kcps}$.
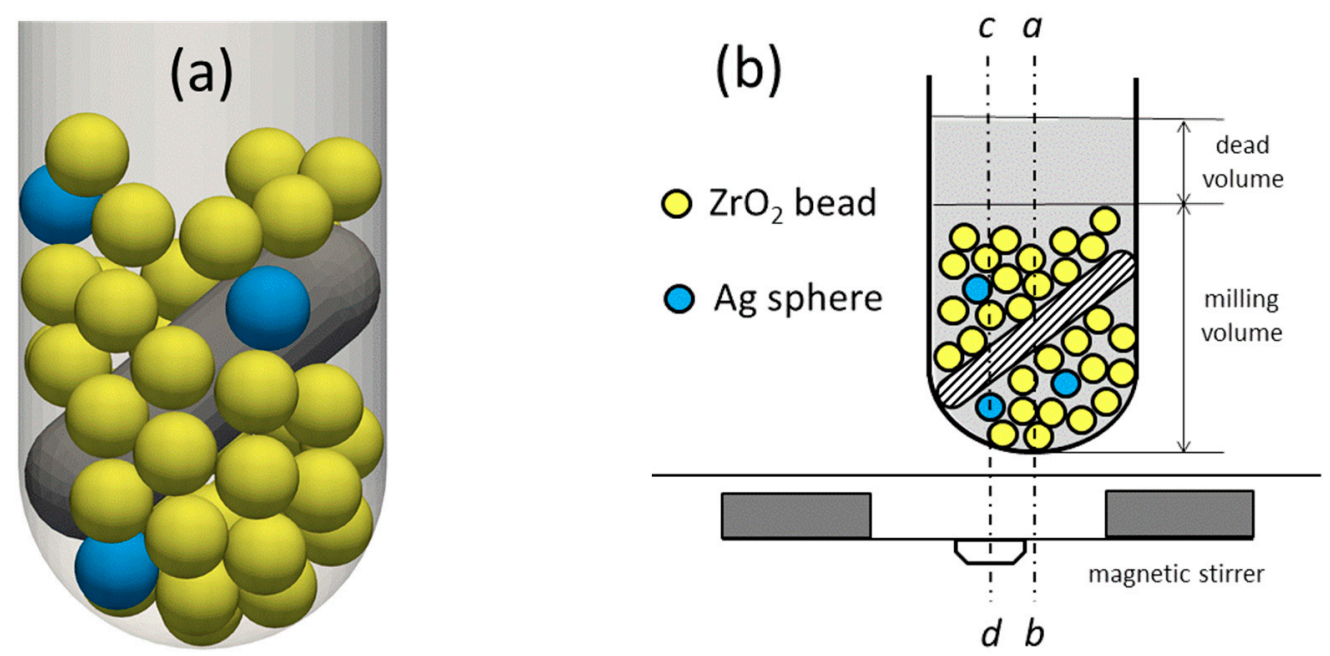

Figure 2. (a) Scheme of the miller assembly with the grey stirring bar in skew position (adapted from the work of [30]); (b) scheme indicating the position of the vessel axis $a b$ with respect to the axis $c d$ of the magnetic stirring disc.

High resolution imaging of the Ag NPs was carried out by transmission electron microscopy (TEM) on an instrument JEM-1011 (Jeol, Japan), operating at $100 \mathrm{kV}$. The solutions of NPs were spread on copper grids coated with carbon films. Compositional analysis of the NPs was performed by energy-dispersive X-ray spectroscopy (EDS) carried out in a scanning electron microscope (SEM) JSM-6490LA (Jeol, Japan), working at $15 \mathrm{kV}$. The solutions of NPs were cast on an aluminium stub and coated with $30 \mathrm{~nm}$ carbon.

\section{Results and Discussion}

In Figure 3, the probability distribution of particle diameters is reported for different groups of capping agents. The curves in the upper left plot (a) refer to three different molecular weights of PVP, whose properties as stabilizer, grafting agent [31], and size-orienting component have been amply recognized and investigated in the literature, both for Ag and for different zerovalent NPs made of non-transition elements [32]. It can be seen that the smallest average particles diameters for non-ionic dispersants are obtained for PVP 10k, namely in the case of shorter polymer chains. Intriguingly, this trend is the opposite of what has been found in the literature concerning the influence of PVP molecular weight on the dimension of Ag NPs synthesized by wet chemical [33] and radiochemical methods [34]. The rationale behind the trend observed here could be found in the mechanism of formation and further stabilization of the NPs in a physical top-down disaggregation process, whose steps differ from those typical of chemically driven bottom-up synthesis methods. In this specific case of non-ionic stabilization, it is supposed that PVP chains of lower molecular weight could more efficiently cover the surface of silver spheres, thus promoting a better stabilization of the clusters detached by shear stress and collision from the metal surface itself. This aspect will be reconsidered at the end of this section. 

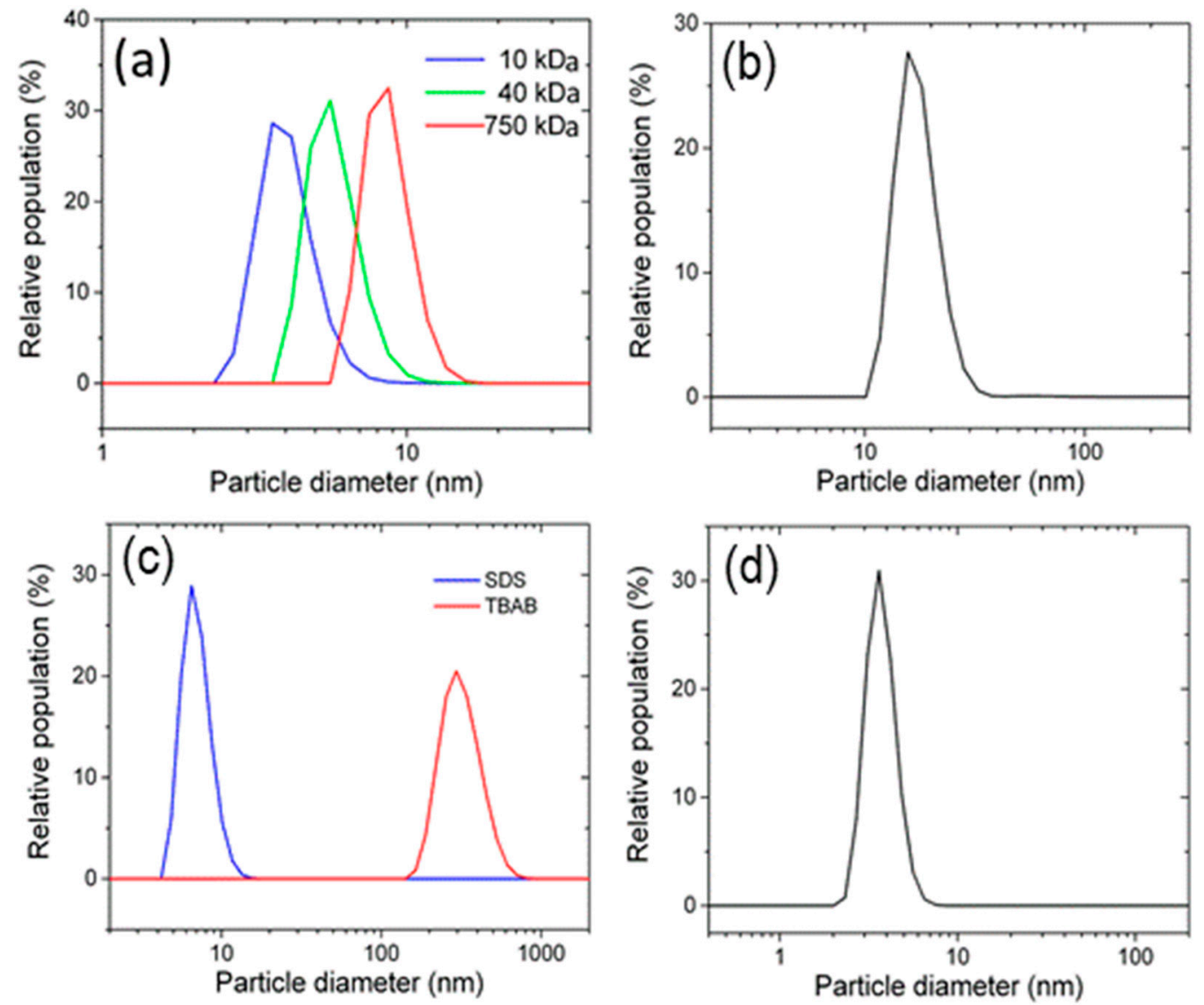

Figure 3. Dynamic light scattering (DLS) measurements of the mean nanoparticle (NP) size resulting from the use of different surfactants. (a) Polyvinyl pyrrolydone (PVP) of various molecular weight (MW); (b) urea (UR); (c) sodium dodecyl sulphate (SDS) and tetrabutylammonium bromide (TBAB); (d) N,N-dimethyldodecylamine N-oxide (DDAO).

In Figure 3b, analogously, the distribution of Ag NPs diameters is reported using urea as capping agent. In this case, Ag NPs with average diameters of $16 \mathrm{~nm}$ were obtained, proving that urea has lower efficiency with respect to PVP of any molecular weight, when lower NPs' diameters are desired.

In Figure 3c, the effect of ionic capping agents is described, showing a markedly higher efficiency of the anionic stabilizer (SDS), giving $6.5 \mathrm{~nm}$ as the average diameter, a value considerably smaller than that obtained with the cationic one (TBAB). In the latter case, only a microsized solid phase was produced with an average diameter of $300 \mathrm{~nm}$, namely larger than those obtained in all other experimental runs.

Figure $3 \mathrm{~d}$ refers to a Zwitterionic capping agent, a proven choice for the synthesis of $\mathrm{Ag}$ nanowires [35] and, in more recent times, for antibacterial surface functionalization by co-deposition of Ag NPs with Zwitterionic polymers [36]. In the present bead milling process, DDAO showed a high efficiency as stabilizer, leading to a dispersion of Ag NPs with an average diameter of $3.5 \mathrm{~nm}$.

Despite the great amount of literature concerning Ag NPs bottom-up synthesis by chemical reduction, a definitive conclusion about the superiority of cationic or anionic stabilizers in that context cannot be easily drawn, as the polarity of the capping agent may even interfere with the thermodynamic and kinetics of the process leading from $\mathrm{Ag}^{+}$to $\mathrm{Ag}^{(0)}$ [37]. Furthermore, as far as Ag NPs stabilization in chemical synthesis is concerned, the following was observed:

- $\quad$ cationic stabilizers have a well-defined shape-orienting effect [38], generally greater than that typical of SDS [37]; 
- $\quad$ electrostatic stabilization by ionic surfactants proved to be more efficient than steric stabilization by non-ionic surfactants [39].

The latter consideration does not seem to hold for the stabilization of Ag NPs produced by a purely physical synthesis method as the one discussed here. In fact, the curves reported in snapshots (a) and (b) of Figure 3, concerning non-ionic capping agents like PVP and urea, lead to average mean diameters being comparable, and in some cases, better than those typical of ionic stabilizers. The lowest average particle diameter obtained with PVP 10k is consistent with the high efficiency of the ketonic group as an electron-pair donor to zerovalent Ag. The effects of different capping agents on Ag NPs average diameters estimated by DLS measurements are reported in Table 2.

Table 2. Average Ag nanoparticles' (NPs') diameters obtained with different capping agents.

\begin{tabular}{cc}
\hline Capping Agent & Ag-NPs' Average Diameter (nm) \\
\hline PVP 10k & 3.6 \\
PVP 40k & 5.6 \\
PVP 750k & 8.7 \\
UR & 16 \\
TBAB & 300 (microparticles) \\
SDS & 6.5 \\
DDAO & 3.5 \\
\hline
\end{tabular}

In Figure 4, TEM images of Ag NPs obtained from samples $a$ and $d$ of Table 1 are reported. In left panel, the NPs diameters are statistically higher than those measured by DLS counting for PVP 10k, but many highlighted NPs have diameters $<10 \mathrm{~nm}$, in agreement with the corresponding distribution curve of Figure 3a. In right panel, the three NPs visualized in white circles have diameters $>10 \mathrm{~nm}$, and these values are consistent with the distribution curve of Figure $3 b$ pertaining to UR as capping agent.
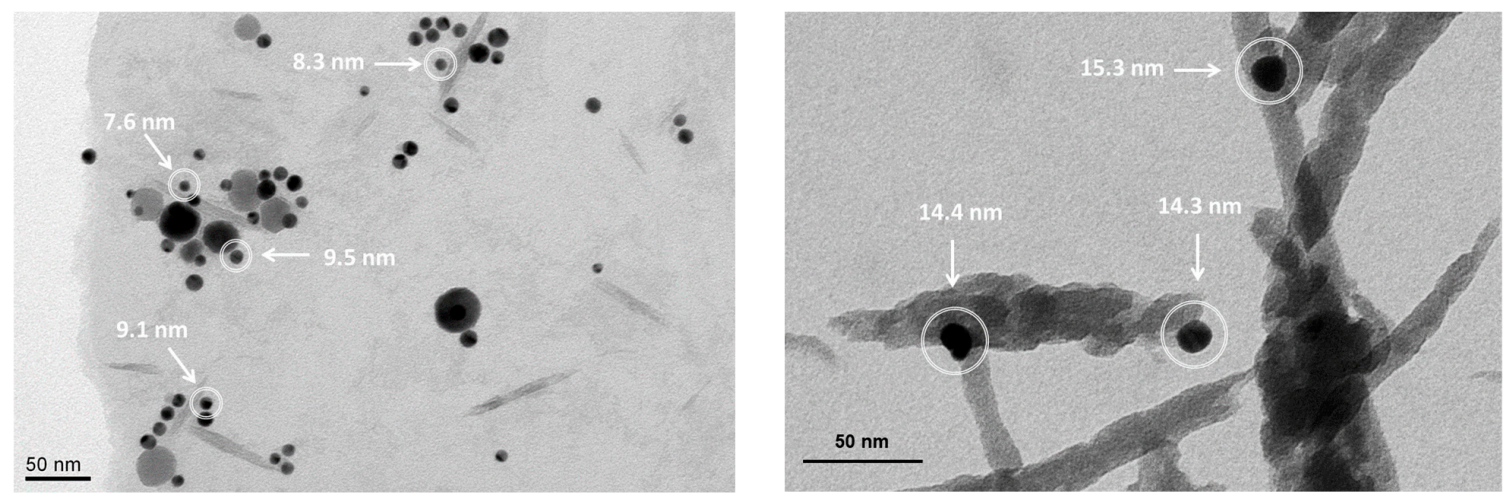

Figure 4. Transmission electron microscopy (TEM) images of samples obtained using PVP 10k and UR as capping agents in the left and right panels, respectively.

Typical qualitative results of EDS compositional analysis are reported in Figure 5, for the case of Ag NPs drop cast from a solution containing UR. From the X-ray background of Bremsstrahlung, Ag peaks clearly emerge, together with C, from both UR and, above all, the SEM drain layer coating and $\mathrm{N}$ from UR. The highest peak is from the Al substrate of the stub, given the deep penetration of the primary electrons in the beam, while residual $\mathrm{O}$ also appears, which is owing to both UR and the oxidized Al stub surface, but is clearly below levels consistent with the presence of Ag oxide. 


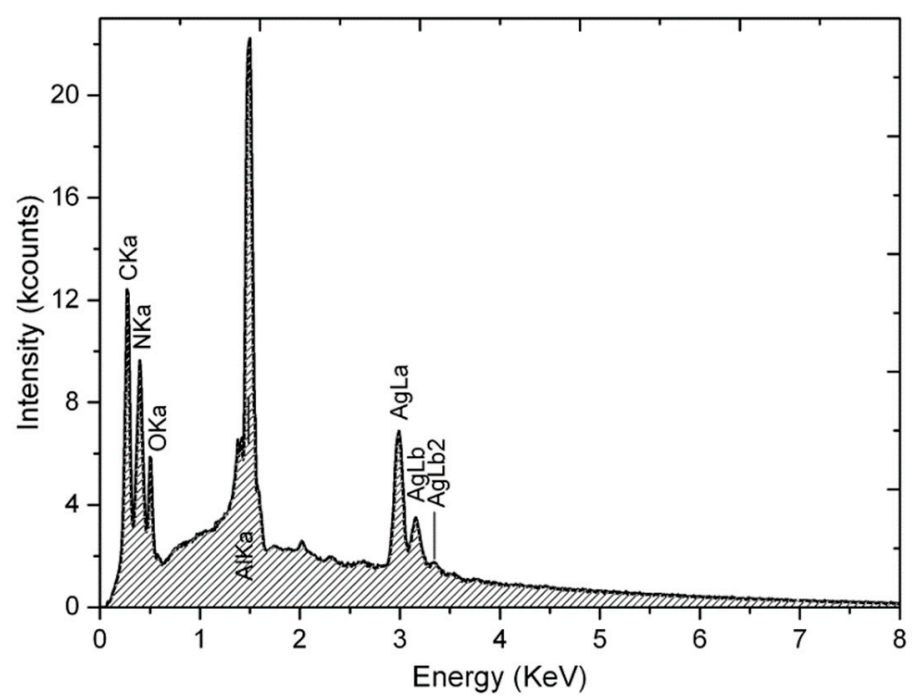

Figure 5. Energy-dispersive X-ray spectroscopy (EDS) spectrum acquired on an aggregate of Ag NPs drop cast from a solution containing UR (scanning electron microscope (SEM) image not shown). The presence of metallic Ag is clearly assessed.

What has been stated up to now in the present section can be summarized in the following points:

- $\quad$ Except for the case of cationic stabilizer (TBAB), Ag NPs of small average diameters $(<16 \mathrm{~nm})$ were obtained in essentially all cases proposed here according to DLS data, with small relative differences between samples, irrespective of the chemical structure and/or functional groups of the corresponding capping agent;

- As a consequence of the above point, this case of top-down disaggregation process describes a new scenario with respect to conventional wet-chemical synthesis, where capping agents differing in chemical composition show remarkable variations in efficiency.

A possible qualitative explanation of such intriguing aspects could be given by taking into account the sequence of dynamic events causing a basic difference between a bottom-up chemical and a top-down physical synthesis process, like that typical of the present study.

In the former, metal ions generate zerovalent atoms, whose concentration grows in time until it reaches a threshold value, triggering the process of aggregation and finally the Ostwald ripening, namely the coarsening mechanism by which larger particles grow by incorporating smaller ones [40]. Moreover, it was observed that surfactants or capping agents are active since the first step of the global process, as they tend to form bonds both with ionic species as well as with nuclei during their initial process of aggregation. [41].

In the latter, an ionic species at the start is present at a negligible concentration related to the thermodynamic equilibria of metal/ion in a solvent, while micro- and nanosized surface elements are progressively detached from the metallic surface by mechanical friction; abrasion; or, more rarely in a low-energy milling, by shock. For these reasons, the mechanisms and the kinetic steps of surface stabilization by capping agents in bottom-up chemical synthesis are deeply different from those occurring in top-down physical synthesis processes, as schematically indicated in Figure 6. The modelling of such different scenarios has been extensively developed in chemical synthesis [42], while it has received very limited attention in bead milling processes until now. 


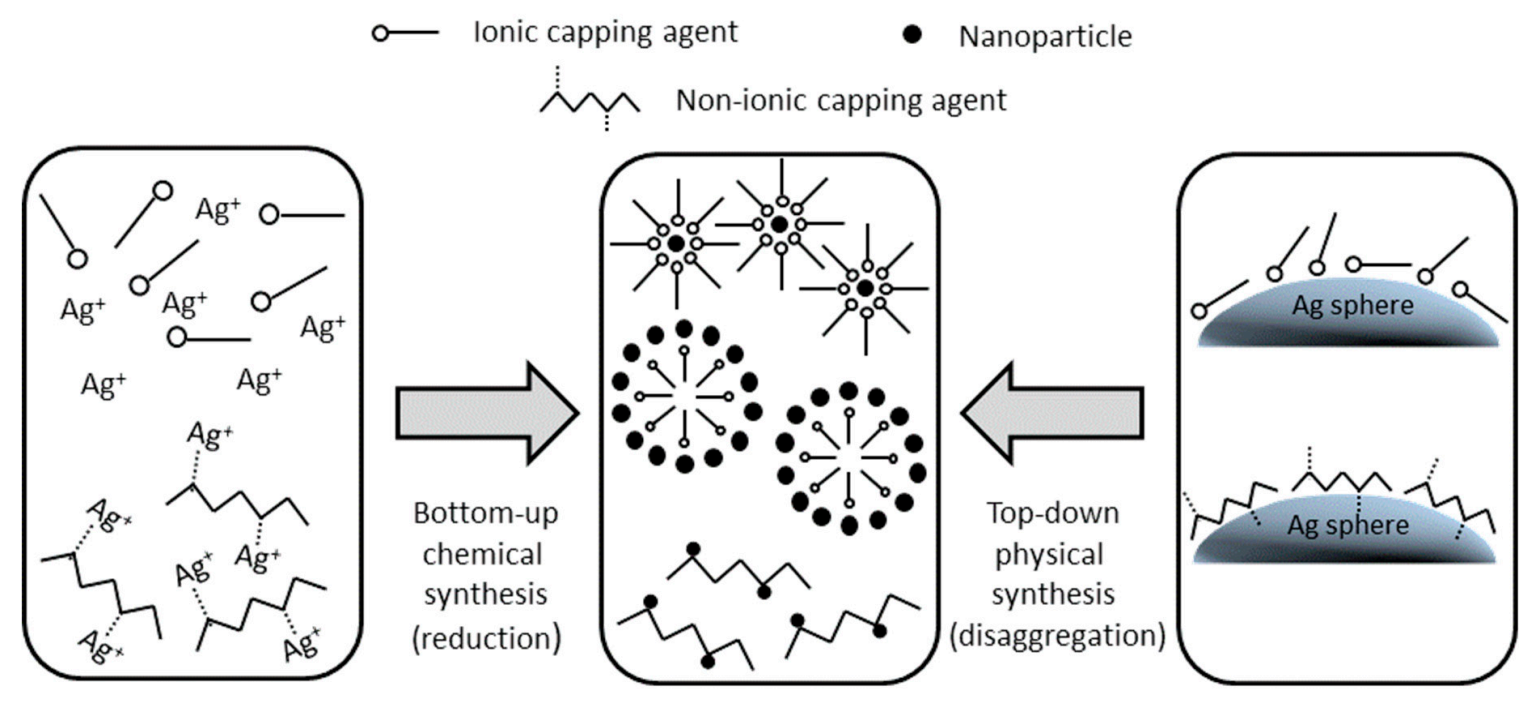

Figure 6. Scheme of different patterns for the synthesis of metal NPs in the case of wet-chemical bottom-up processes and in the case of top-down physical disaggregation by wet bead milling (the present study).

As anticipated in the Introduction, milling processes for NPs manufacturing are currently in the hotspot for their sustainability and versatility in drug preparation [43] and in mechanochemical synthesis [44]. Ogi et al. [24] authored an interesting review paper containing a systematic classification of machining processes for the production of NPs dispersions by milling methods. Among them, wet bead-milling continuous processes are extensively analyzed and many details concerning different technical solutions are presented, essentially differing from one another about the realization of the rotor pin and of the centrifugal separator between beads and NPs. In particular, the authors pointed out the difference between media-less and media-assisted methods in top-down disaggregation. Among the latter ones, it is worth reminding that low-energy bead-milling/bead-stirring apparatuses are generally used to decrease the diameter of particles already belonging to a micron or even submicron range. This amounts to saying that such processes are essentially designed to disaggregate NP agglomerates [45], thereby releasing primary particles, and this explains why bead millers are fed with liquids containing suspended particles, even forming nanodispersions at the start [46], as well known in pharmaceutical science [47]. A crucial aspect in these processes is the establishment of an optimal matching between the size of the solid phase feeding the miller and the diameter of the beads carrying out the disaggregation, as a relatively large inter-bead space at a close-packing configuration may lead to a dramatic loss of milling efficiency [48]. In a previous work of ours [49], a nanophase made of primary particles was produced in a two-pot process, where agglomerated crystals of zerovalent bismuth obtained by chemical cementation underwent a wet bead-milling to reach a final state of stable nanometric dispersion.

On the opposite, studies proposing a one-pot bead milling process where NPs are produced directly starting from a bulky solid like metal spheres of millimetric dimension are quite uncommon in the literature. The present work is an example of such an approach, with a magnetically-driven motion of both precursor and milling spheres. In a somewhat different context, Romero et al. [50] proposed a miniaturized and magnetically driven wet bead miller to comminute an initially microcrystalline phase in a batch process, with beads' diameters spanning in the range of $0.05-0.6 \mathrm{~mm}$. Their results essentially confirmed the well-known rule of thumb, stating that the minimum achievable final diameter of particles may reach $1 / 1000$ of the bead diameter in wet bead milling equipment.

In the present non-conventional bead milling technique, the aforementioned rule seems to be violated by more than two orders of magnitude. In fact, in all cases but one of Ag disaggregation carried out in aqueous solvent, whatever the capping agent, the nanoparticle diameters are smaller than 30 
$\mathrm{nm}$, a value corresponding to a $1 / 10^{5}$ ratio between $\mathrm{Ag} \mathrm{NP}$ and bead diameters. A possible explanation could be given taking into account the following points, which highlight the main differences between the present disaggregation method and the standard bead milling processes:

- The aforementioned rule of " $1 / 1000$ " has proven its validity in the presence of an initial solid phase made of agglomerated crystals or particles having microsized dimensions. The present case, instead, concerns a compact homogeneous solid phase of a bulky metal.

- Unlike the scheme of Romero et al. [50], the present disaggregation process occurs primarily at the bottom of the vessel, as depicted in Figure 2b, owing to the weight of both beads and metal spheres. In this volume, the metal-bead friction is particularly active, leading to a metal surface disaggregation with release of micro- and nanoparticles. Here, the stirring bar turns along the generatrix of a cone, creating zones of null collision beside zones with a high probability of collisions among spheres, thus enhancing the velocity gradients and ultimately the shear stresses on Ag spheres.

- The dead volume at the top of the vessel is now beneficial, acting like a sort of escaping zone for the nanoparticles, subtracting them from the milling volume and thus reducing their probability of sintering and reagglomeration. This new aspect might explain why, in the present study, NPs of considerably small diameters were obtained in almost all cases, irrespective of the capping agent used.

Mc Mahon et al. [51] addressed the problem of whether a capping agent directly acts on the surface of a bulk solid subject to disaggregation. They synthesized $\mathrm{Al}, \mathrm{Cu}$, and Fe NPs in a planetary miller using different solvents and surfactants. The highest NPs/microparticles mass ratio was obtained using solvent forming bonds or having affinities with the metal surface. More intriguingly, oleic acid acted as one of the most efficient capping agents in promoting the formation of metal NPs, despite its lubricating properties. It was conjectured that a basic role in such a process is played by the adsorption of solvent or surfactant on the metal surface, whose free energy is lowered, thus enhancing its chemisorption-induced decohesion and finally its disaggregation [52]. Admittedly, in agreement with the latter consideration, analogous mechanism can be invoked in this case of low-energy disaggregation, to explain an unexpected synthesis of metal NPs starting from a bulky solid.

\section{Conclusions}

A bead-milling process for the synthesis of Ag NPs using Ag spheres as source material was proposed. In terms of practical relevance, such a method may represent an alternative to chemical routes in the pharmaceutical industry whenever the preparation of antiseptic/antibacterial solutions is required in emergency situations, thanks to the small scale and portability of the experimental setup described here.

From a methodological point of view, the key aspects of the present work can be summarized as follows:

- $\quad$ Ag NPs were obtained by a simple, non-conventional, and cost-effective bead milling apparatus based on a low-energy disaggregation process. The miller does not need a moving shaft of complex shape with gas-tight fittings, neither built-in centrifugal separators for milling bead recovery, nor additional temperature control devices, with a considerable simplification of the miller setup, compared with the usually tricky bead-milling solutions.

- The requirements of eco-compatibility are met in this simple top-down physical process where a precursor at its elemental state is used, as no toxic or noxious chemical reactant is needed. The apparatus is scalable, within the limits imposed by the efficiency of a magnetic stirrer in larger plants.

- Excluding the case concerning a cationic stabilizer, Ag NPs with average diameters $<16 \mathrm{~nm}$ were obtained with all the other capping agents, proving the global reliability of the process in terms of quality of the final product. 
- Tuning the shape of NPs produced by the present set-up is not considered so far. It can be conjectured that temperature may have an important role on the surface adsorption of each capping agent on the bulk metal surface, thereby conditioning the shape of the as-made NPs.

- To the best of our knowledge, this is one of the very few wet bead-milling processes where the diameter of the milled phase gets nanosized by means of the milling media and precursor both belonging to a millimetric scale.

A future development of such process will be aimed at synthesizing zerovalent NPs of non-noble metals for toxic waste decontamination.

Author Contributions: Conceptualization, A.P.R.; methodology A.P.R. and B.F.; validation, M.V.; investigation, M.S. and M.V.; resources, B.F. and M.F.; data curation, A.P.R. and M.S.; writing-original draft preparation, A.P.R.; writing—review and editing, A.P.R., M.S., and M.V.; visualization, M.S.; supervision, M.F. and B.F.; project administration, M.V.; funding acquisition, M.V. All authors have read and agreed to the published version of the manuscript.

Funding: This research received no external funding.

Conflicts of Interest: The authors declare no conflict of interest.

\section{Abbreviations}

The following abbreviations are used in this manuscript:

cP centiPoise

cps counts per second

Da Dalton

DDAO N,N-dimethyldodecylamine N-oxide

DLS dynamic light scattering

EDS energy dispersive X-ray spectroscopy

MW molecular weight

NG nitrogen-doped graphene

NPs nanoparticles

PVP 10k polyvinyl pyrrolydone with $10 \mathrm{kDa}$ molecular weight

PVP 40k polyvinyl pyrrolydone with $40 \mathrm{kDa}$ molecular weight

PVP 750k polyvinyl pyrrolydone with $750 \mathrm{kDa}$ molecular weight

PTFE polytetrafluoro ethylene

SDS sodium dodecyl sulphate

SEM scanning electron microscopy

TBAB tetrabutylammonium bromide

TEM transmission electron microscopy

UR urea

\section{References}

1. Teoh, W.Y.; Amal, R.; Mädler, L. Flame spray pyrolysis: An enabling technology for nanoparticles design and fabrication. Nanoscale 2010, 2, 1324-1347. [CrossRef]

2. Reverberi, A.P.; Salerno, M.; Lauciello, S.; Fabiano, B. Synthesis of copper nanoparticles in ethylene glycol by chemical reduction with vanadium (+2) salts. Materials 2016, 9, 809. [CrossRef] [PubMed]

3. Fabiano, B.; Reverberi, A.P.; Varbanov, P.S. Safety opportunities for the synthesis of metal nanoparticles and short-cut approach to workplace risk evaluation. J. Clean. Prod. 2019, 209, 297-308. [CrossRef]

4. Vocciante, M.; De Folly D’Auris, A.; Finocchi, A.; Tagliabue, M.; Bellettato, M.; Ferrucci, A.; Reverberi, A.P.; Ferro, S. Adsorption of ammonium on clinoptilolite in presence of competing cations: Investigation on groundwater remediation. J. Clean. Prod. 2018, 198, 480-487. [CrossRef]

5. Chen, X.; Lim, T.T. Highly-efficient peroxymonosulfate activation for sulfacetamide degradation over nitrogen-functionalized graphene: The effect of thermal annealing temperature on reactive functional groups. Chem. Eng. Trans. 2019, 73, 145-150. 
6. Toccafondi, C.; Dante, S.; Reverberi, A.P.; Salerno, M. Biomedical applications of anodic porous alumina. Curr. Nanosci. 2015, 11, 572-580. [CrossRef]

7. Fabiano, B.; Pistritto, F.; Reverberi, A.; Palazzi, E. Ethylene-air mixtures under flowing conditions: A model-based approach to explosion conditions. Clean Technol. Environ. Policy 2015, 17, 1261-1270. [CrossRef]

8. Zhang, S.; Zhang, C.; Liu, M.; Huang, R.; Su, R.; Qi, W.; He, Z. Dechlorination of p-chlorophenol by Fe-Pd nanoparticles promoted by poly ( $\gamma$-glutamic acid)-Dopamine composite. Chem. Eng. Trans. 2018, 70, 2095-2100.

9. Lu, H.; Tian, B.; Wang, J.; Hao, H. Montmorillonite-supported Fe/Ni bimetallic nanoparticles for removal of Cr(VI) from wastewater. Chem. Eng. Trans. 2017, 60, 169-174.

10. De Souza, C.D.; Ribeiro Nogueira, B.; Rostelato, M.E.C.M. Review of the methodologies used in the synthesis gold nanoparticles by chemical reduction. J. Alloys Compd. 2019, 798, 714-740. [CrossRef]

11. Stefaniuk, M.; Oleszczuk, P.; Ok, Y.S. Review on nano zerovalent iron (nZVI): From synthesis to environmental applications. Chem. Eng. J. 2016, 287, 618-632. [CrossRef]

12. Rodrigues, T.S.; Zhao, M.; Yang, T.H.; Gilroy, K.D.; Da Silva, A.G.M.; Camargo, P.H.C.; Xia, Y. Synthesis of colloidal metal nanocrystals: A comprehensive review on the reductants. Chem. Eur. J. 2018, 24, 16944-16963. [CrossRef] [PubMed]

13. Matsumoto, M.; Kano, H.; Suzuki, M.; Katagiri, T.; Umeda, Y.; Fukushima, S. Carcinogenicity and chronic toxicity of hydrazine monohydrate in rats and mice by two-year drinking water treatment. Regul. Toxicol. Pharmacol. 2016, 76, 63-73. [CrossRef] [PubMed]

14. Promentilla, M.A.B.; Janairo, J.I.B.; Yu, D.E.C. A green chemistry-based decision modelling approach for optimal selection of nanomaterial's synthesis method. Chem. Eng. Trans. 2017, 61, 241-246.

15. Reverberi, A.P.; Vocciante, M.; Lunghi, E.; Pietrelli, L.; Fabiano, B. New trends in the synthesis of nanoparticles by green methods. Chem. Eng. Trans. 2017, 61, 667-672.

16. Reverberi, A.P.; Varbanov, P.S.; Vocciante, M.; Fabiano, B. Bismuth oxide-related photocatalysts in green nanotechnology: A critical analysis. Front. Chem. Sci. Eng. 2018, 12, 878-892. [CrossRef]

17. Chew, K.H.; Klemeš, J.J.; Alwi, S.R.W.; Manan, Z.A.; Reverberi, A.P. Total site heat integration considering pressure drops. Energies 2015, 8, 1114-1137. [CrossRef]

18. Sivashanmugam, P. Application of Nanofluids in Heat Transfer. In An Overview of Heat Transfer Phenomena; Kazi, S.N., Ed.; BoD: Rijeka, Croatia, 2012; pp. 411-441.

19. Nagar, N.; Devra, V. Green synthesis and characterization of copper nanoparticles using Azadirachta indica leaves. Mater. Chem. Phys. 2018, 213, 44-51. [CrossRef]

20. Abdullah, R.M.; Aziz, S.B.; Mamand, S.M.; Hassan, A.Q.; Hussein, S.A.; Kadir, M.F.Z. Reducing the crystallite size of spherulites in PEO-based polymer nanocomposites mediated by carbon nanodots and Ag nanoparticles. Nanomaterials 2019, 9, 874. [CrossRef]

21. Korir, D.K.; Gwalani, B.; Joseph, A.; Kamras, B.; Arvapally, R.K.; Omary, M.A.; Marpu, S.B. Facile photochemical syntheses of conjoined nanotwin gold-silver particles within a biologically-benign chitosan polymer. Nanomaterials 2019, 9, 596. [CrossRef]

22. Yang, L.; Chu, D.; Wang, L.; Ge, G.; Sun, H. Facile synthesis of porous flower-like $\mathrm{SrCO}_{3}$ nanostructures by integrating bottom-up and top-down routes. Mater. Lett. 2016, 167, 4-8. [CrossRef]

23. Vocciante, M.; Finocchi, A.; D'Auris, A.F.; Conte, A.; Tonziello, J.; Pola, A.; Reverberi, A.P. Enhanced oil spill remediation by adsorption with interlinked multilayered graphene. Materials 2019, 12, 2231. [CrossRef] [PubMed]

24. Ogi, T.; Zulhijah, R.; Iwaki, T.; Okuyama, K. Recent progress in nanoparticle dispersion using bead mill. KONA Powder Part. J. 2017, 34, 3-23. [CrossRef]

25. Ali, M.E.; Ullah, M.; Maamor, A. Surfactant-assisted ball-milling: A simple top-down approach for the synthesis of controlled structure nanoparticle. Adv. Mater. Res. 2014, 832, 356-361. [CrossRef]

26. Ullah, M.; Ali, M.E.; Hamid, S.B.A. Surfactant-assisted ball milling: A novel route to novel materials with controlled nanostructure-A review. Rev. Adv. Mater. Sci. 2014, 37, 1.

27. Kumar, N.; Biswas, K.; Gupta, R.K. Green synthesis of Ag nanoparticles in large quantity by cryomilling. RSC Adv. 2016, 6, 111380-111388. [CrossRef]

28. Yamamoto, Y.; Soda, R.; Kano, J.; Saito, F. DEM simulation of bead motion during wet bead milling using an enlarged particle model. Int. J. Miner. Process. 2012, 114-117, 93-99. [CrossRef] 
29. Dang, T.M.D.; Le, T.T.T.; Fribourg-Blanc, E.; Dang, M.C. Synthesis and optical properties of copper nanoparticles prepared by a chemical reduction method. Adv. Nat. Sci. Nanosci. Nanotechnol. 2011, 2, 015009. [CrossRef]

30. Vocciante, M.; Trofa, M.; D'Avino, G.; Reverberi, A.P. Nanoparticles synthesis in wet-operating stirred media: Preliminary investigation with DEM simulations. Chem. Eng. Trans. 2019, 73, 31-36.

31. Grytsenko, O.; Gajdoš, I.; Spišák, E.; Krasinskyi, V.; Suberlyak, O. Novel Ni/pHEMA-gr-PVP composites obtained by polymerization with simultaneous metal deposition: Structure and properties. Materials 2019, 12, 1956. [CrossRef]

32. Reverberi, A.P.; Vocciante, M.; Salerno, M.; Caratto, V.; Fabiano, B. Bi nanoparticles synthesis by a bottom-up wet chemical process. Chem. Eng. Trans. 2019, 73, 283-288.

33. Ummartyotin, S.; Bunnak, N.; Juntaro, J.; Sain, M.; Manuspiya, H. Synthesis of colloidal silver nanoparticles for printed electronics. Comptes Rendus Chim. 2012, 15, 539-544. [CrossRef]

34. Jovanović, Z.; Radosavljević, A.; Šiljegović, M.; Bibić, N.; Mišković-Stanković, V.; Kačarević-Popović, Z. Structural and optical characteristics of silver/poly(N-vinyl-2-pyrrolidone) nanosystems synthesized by $\gamma$-irradiation. Radiat. Phys. Chem. 2012, 81, 1720-1728. [CrossRef]

35. Matsune, H.; Kuramitsu, Y.; Takenaka, S.; Kishida, M. Chemical synthesis of silver nanowires using N,N-dimethyldodecylamine oxide. Chem. Lett. 2010, 39, 717-719. [CrossRef]

36. Xie, Y.; Chen, S.; Zhang, X.; Shi, Z.; Wei, Z.; Bao, J.; Zhao, W.; Zhao, C. Engineering of tannic acid inspired antifouling and antibacterial membranes through co-deposition of zwitterionic polymers and $\mathrm{Ag}$ nanoparticles. Ind. Eng. Chem. Res. 2019, 58, 11689-11697. [CrossRef]

37. Khan, Z.; Bashir, O.; Hussain, J.I.; Kumar, S.; Ahmad, R. Effects of ionic surfactants on the morphology of silver nanoparticles using Paan (Piper betel) leaf petiole extract. Colloids Surf. B Biointerfaces 2012, 98, 85-90. [CrossRef]

38. Al-Thabaiti, S.A.; Obaid, A.Y.; Hussain, S.; Khan, Z. Shape-directing role of cetyltrimethylammonium bromide on the morphology of extracellular synthesis of silver nanoparticles. Arab. J. Chem. 2015, 8, 538-544. [CrossRef]

39. Shah, V.; Bharatiya, B.; Mishra, M.K.; Ray, D.; Shah, D.O. Molecular insights into sodium dodecyl sulphate mediated control of size for silver nanoparticles. J. Mol. Liq. 2019, 273, 222-230. [CrossRef]

40. Wu, Z.; Yang, S.; Wu, W. Shape control of inorganic nanoparticles from solution. Nanoscale 2016, 8, 1237-1259. [CrossRef]

41. De, S.; Mandal, S. Surfactant-assisted shape control of copper nanostructures. Colloids Surf. A Physicochem. Eng. Asp. 2013, 421, 72-83. [CrossRef]

42. Amirjani, A.; Haghshenas, D.F. Modified Finke-Watzky mechanisms for the two-step nucleation and growth of silver nanoparticles. Nanotechnology 2018, 29, 505602. [CrossRef] [PubMed]

43. Nakach, M.; Authelin, J.R.; Perrin, M.A.; Lakkireddy, H.R. Comparison of high pressure homogenization and stirred bead milling for the production of nano-crystalline suspensions. Int. J. Pharm. 2018, 547, 61-71. [CrossRef] [PubMed]

44. Xu, C.; De, S.; Balu, A.M.; Ojeda, M.; Luque, R. Mechanochemical synthesis of advanced nanomaterials for catalytic applications. Chem. Commun. 2015, 51, 6698-6713. [CrossRef] [PubMed]

45. Joni, I.M.; Ogi, T.; Iwaki, T.; Okuyama, K. Synthesis of a colorless suspension of $\mathrm{TiO}_{2}$ nanoparticles by nitrogen doping and the bead-mill dispersion process. Ind. Eng. Chem. Res. 2013, 52, 547-555. [CrossRef]

46. Tahara, T.; Imajyo, Y.; Nandiyanto, A.B.D.; Ogi, T.; Iwaki, T.; Okuyama, K. Low-energy bead-milling dispersions of rod-type titania nanoparticles and their optical properties. Adv. Powder Technol. 2014, 25, 1492-1499. [CrossRef]

47. Nakach, M.; Authelin, J.R.; Agut, C. New approach and practical modelling of bead milling process for the manufacturing of nanocrystalline suspensions. J. Pharm. Sci. 2017, 106, 1889-1904. [CrossRef] [PubMed]

48. Bead Mill-Grinding \& Dispersing. Available online: http://www.hiroshimamm-chemtech.com/en/ knowledge/detail01/ (accessed on 31 October 2019).

49. Reverberi, A.P.; Varbanov, P.S.; Lauciello, S.; Salerno, M.; Fabiano, B. An eco-friendly process for zerovalent bismuth nanoparticles synthesis. J. Clean. Prod. 2018, 198, 37-45. [CrossRef]

50. Romero, G.B.; Keck, C.M.; Müller, R.H. Simple low-cost miniaturization approach for pharmaceutical nanocrystals production. Int. J. Pharm. 2016, 501, 236-244. [CrossRef] 
51. Mc Mahon, B.V.; Perez, J.P.L.; Yu, J.; Boatz, J.A.; Anderson, S.L. Synthesis of nanoparticles from malleable and ductile metals using powder-free, reactant-assisted mechanical attrition. Appl. Mater. Interfaces 2014, 6, 19579-19591. [CrossRef]

52. Gavriljuk, V.G.; Teus, S.M.; Shanina, B.D.; Konchits, A.A. On the nature of similarity in embrittlement of metals by hydrogen and surfactants. Mater. Sci. Eng. Int. J. 2017, 1, 70-79. 